\title{
Unexpected boost of thermoelectric performance by magnetic nanoparticles
}

\author{
Jiangyu $\mathrm{Li}^{1,2}$
}

Thermoelectric materials are capable of converting heat into electricity and vice versa, and thus they are attractive in waste heat recovery and solid-state thermal management. Nevertheless, thermoelectric technologies are only limited to niche applications so far, for example in space exploration, since it is rather challenging to enhance their conversion efficiency. The performance of thermoelectric materials is usually characterized by their figure of merit $Z T=\left[\sigma \alpha^{2} /\left(\kappa_{e}+\kappa_{l}\right)\right] T$, and it is well known that high electric conductivity $\sigma$ and Seebeck coefficient $\alpha$ and low thermal conductivity $\kappa$ impose different and often competing requirements [1]. For example, the electronic contribution to the thermal conductivity $\kappa_{l}$ is proportional to $\sigma$. As a result, over the last 30 years of $20^{\text {th }}$ century, very little advance has been made in thermoelectric figure of merit.

With the turn of new century, we have witnessed rapid increase in thermoelectric performance in the past two decades and ZT of many materials has doubled, thanks to the emergence of nanomaterials and better understanding of thermoelectric transport processes [2,3]. Such an enhancement has been accomplished mainly by two strategies. One is to reduce phonon contribution to thermal conductivity $\kappa_{l}$ through phonon scattering at interfaces of nanostructured materials $[4,5]$, and the other is to optimize electric conductivity and Seebeck coefficient through band engineering [6,7]. In order to enhance thermoelectric performance, conventional wisdom is that magnetic impurities should be avoided since they may degrade electric transport properties. A couple of papers recently published by a team led by Prof. Qingjie Zhang at Wuhan University of Technology, however, have challenged this conventional thought $[8,9]$.

The initial success was made by embedding hard magnetic $\mathrm{BaFe}_{12} \mathrm{O}_{19}$ nanoparticles into thermoelectric
$\mathrm{Ba}_{0.3} \mathrm{In}_{0.3} \mathrm{Co}_{4} \mathrm{Sb}_{12}$ matrix, which was reported in Nature Nanotechnology last year [8]. The team discovered that the thermoelectric performance degradation in the intrinsic excitation regime can be suppressed by $\mathrm{BaFe}_{12} \mathrm{O}_{19}$ nanoparticles. In addition to usual phonon scattering by the nanoparticles, the team demonstrated that magnetic nanoparticles serve as electron repository, which can trap electrons via Lorenz force at lower temperature and release them after ferromagnetic to paramagnetic transition beyond Curie temperature. When soft magnetic nanoparticles are used instead, there is another ferromagnetic to superparamagnetic transition. The thermal fluctuation of magnetic moment in the superparamagnetic state provides extra phonon scattering to reduce thermal conductivity (Fig. 1a), and they reported this observation recently in Nature using $\mathrm{Ba}_{0.3} \mathrm{In}_{0.3} \mathrm{Co}_{4} \mathrm{Sb}_{12}$ as matrix and soft magnetic cobalt nanoparticles as inclusions [9]. Furthermore, due to the difference in the work functions between matrix and inclusions, the cobalt nanoparticles inject electrons into the matrix, increasing the carrier concentration and electric conductivity (Fig. 1b). The multiple scattering of electrons, similar to Kondo effect induced by the superparamagnetism, and the filtering of low energy electrons enhance Seebeck coefficient (Fig. 1c). As a result, these three concurrent microscopic mechanisms induced by soft magnetic nanoparticles simultaneously increase the electric conductivity and Seebeck coefficient and decrease the thermal conductivity, resulting in 32\% enhancement in $Z T$.

These beautiful mechanisms have potential applications in other thermoelectric materials, and thus inaugurate a new approach for thermoelectric optimization. However, the experimental demonstration of local correlations between magnetism and thermoelectric transport is challenging. The advanced scanning probe microscopy

\footnotetext{
${ }^{1}$ Shenzhen Key Laboratory of Nanobiomechanics, Shenzhen Institutes of Advanced Technology, Chinese Academy of Sciences, Shenzhen 518055, China

${ }^{2}$ Department of Mechanical Engineering, University of Washington, Seattle, 98195-2600, USA Email: jjli@uw.edu
} 

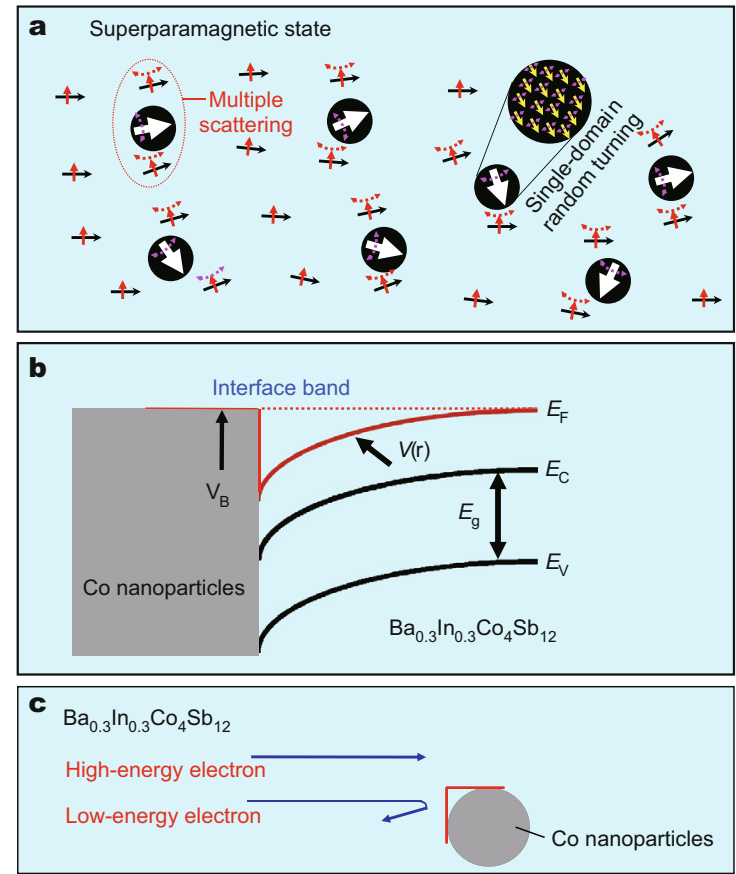

Figure 1 (a) The thermal fluctuation of magnetic moment in the superparamagnetic state of nanoparticles provides extra phonon scattering to reduce thermal conductivity; (b) the cobalt nanoparticles inject electrons into the matrix, increasing the carrier concentration and electric conductivity; (c) the presence of interface potential causes energy-dependent scattering of electrons and the filtering of low energy electrons enhance Seebeck coefficient. This picture is from reference [9]. Copyright 2017 Macmillan Publishers Limited.

techniques have the potential to establish such correlation at the nanoscale, by measuring magnetic states and transport properties near a magnetic nanoparticle in a thermoelectric matrix. Recent study of scanning thermal microscopy has revealed one-to-one correspondence between microscopic composition and local thermal conductivity in a three-phase filled skutterudites at the nanoscale [10], and combining with magnetic force microscopy, it could reveal how the magnetic states in a nanoparticle influence the local thermoelectric performance.

Received 21 September 2017; accepted 21 September 2017; published online 28 September 2017

1 Snyder GJ, Toberer ES. Complex thermoelectric materials. Nat Mater, 2008, 7: 105-114

2 Heremans JP, Dresselhaus MS, Bell LE, et al. When thermoelectrics reached the nanoscale. Nat Nanotech, 2013, 8: 471-473

3 Xie Y. Mosaic crystals leading a new route to achieve ultrahigh thermoelectric performance. Sci China Mater, 2015, 58: 431-432

4 Poudel B, Hao Q, Ma Y, et al. High-thermoelectric performance of nanostructured bismuth antimony telluride bulk alloys. Science, 2008, 320: 634-638

5 Farooq MU, Butt S, Gao K, et al. Pronounced effect of ZnTe nanoinclusions on thermoelectric properties of $\mathrm{Cu}_{2-x} \mathrm{Se}$ chalcogenides. Sci China Mater, 2016, 59: 135-143

6 Pei Y, Shi X, LaLonde A, et al. Convergence of electronic bands for high performance bulk thermoelectrics. Nature, 2011, 473: 66-69

7 Zhao LD, Tan G, Hao S, et al. Ultrahigh power factor and thermoelectric performance in hole-doped single-crystal SnSe. Science, 2016, 351: 141-144

8 Zhao W, Liu Z, Wei $\mathrm{P}$, et al. Magnetoelectric interaction and transport behaviours in magnetic nanocomposite thermoelectric materials. Nat Nanotech, 2017, 12: 55-60

9 Zhao W, Liu Z, Sun Z, et al. Superparamagnetic enhancement of thermoelectric performance. Nature, 2017, 549: 247-251

10 Esfahani EN, Ma F, Wang S, et al. Quantitative nanoscale mapping of three-phase thermal conductivities in filled skutterudites via scanning thermal microscopy. Natl Sci Rev, 2017, doi: 10.1093/ nsr/nwx074 